\title{
Avoiding malpractice while treating depression in pregnant women
}

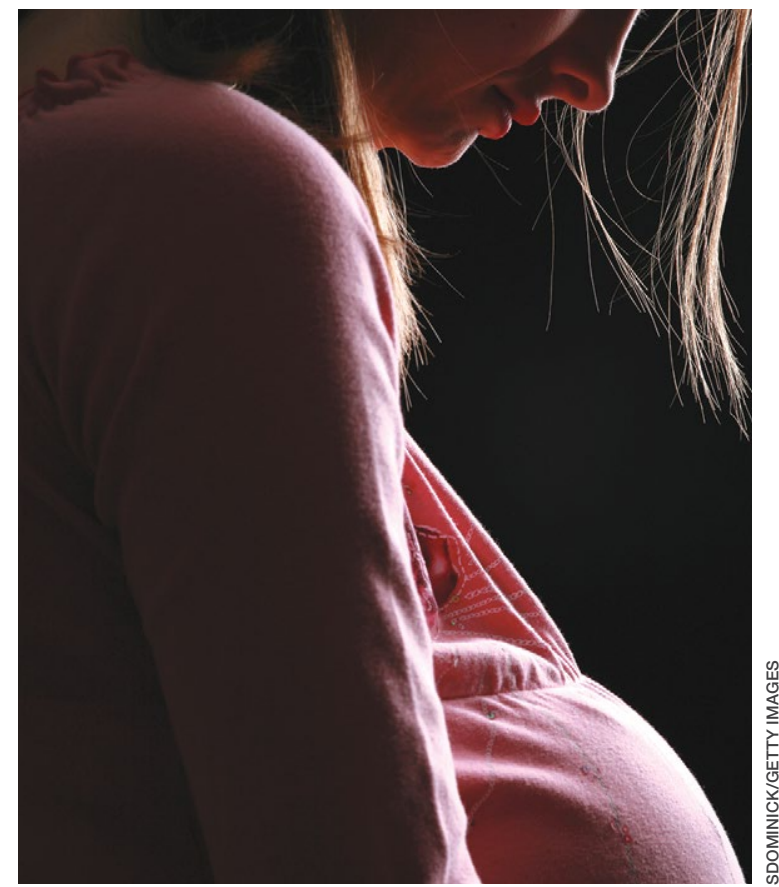

\section{Susan Hatters Friedman, MD}

The Phillip Resnick Professor of Forensic Psychiatry

Professor of Reproductive Biology and Pediatrics

Adjunct Professor of Law

Case Western Reserve University

Cleveland, Ohio

\section{Ryan C.W. Hall, MD}

Associate Professor

University of Central Florida

Orlando, Florida

Affiliated Associate Professor

University Of South Florida

Tampa, Florida

Adjunct Faculty

Barry Law School

Orlando, Florida

\section{Consider the risks of untreated mental illness as well as the risks of antidepressant exposure}

$\mathrm{M}$ any physicians have seen advertisements that encourage women who took an antidepressant while they were pregnant and had a negative outcome to contact a law firm. These ads could make patients more reluctant to take prescribed antidepressants, and psychiatrists more hesitant to prescribe necessary medications during pregnancy - which is a disservice to the mother and child.

More recently, several headline-grabbing studies appeared to suggest that there is an increased risk to infants who are exposed to antidepressants prenatally. Unfortunately, many patients do not understand that replication of these studies is often lacking, and methodological and confounding issues abound. All of this makes it difficult for patients and their families to know if they should take an antidepressant during pregnancy, and for psychiatrists to know what to discuss about the risks and benefits of various antidepressants during pregnancy. This article reviews the rationale for treatment of depression in pregnancy; the risks of untreated depression in pregnancy, as well as the potential risks of medication; ethical issues in the treatment of depression in pregnancy; the limitations of available research; and best approaches for practice.

\section{Risks of untreated depression in pregnancy}

Pregnant women may have misconceptions about treatment during pregnancy, and psychiatrists often are hesitant to treat pregnant women. However, the risks of untreated depression

The authors report no financial relationships with any companies whose products are mentioned in this article, or with manufacturers of competing products.

doi: 10.12788/cp.0154 
during pregnancy are even greater than the risks of untreated depression at other points in a woman's life. In addition to general psychiatric risks seen in depression, pregnant women may experience other issues, such as preeclampsia and liver metabolism changes. ${ }^{1-2}$ Risks to the fetus related to untreated or partially treated mental health concerns include poor prenatal care related to poor self-care, an increased risk of exposure to illicit substances or alcohol related to "self-medication," preterm delivery, and low birthweight (Table 13-8). Further risks for an infant of a mother with untreated depression include decreased cognitive performance and poor bonding with poor stress adaptation. ${ }^{5,6}$ Thus, appropriate treatment of depression is even more important during pregnancy than at other times of life.

\section{Potential risks of treating depression in pregnancy}

When prescribing psychotropic medications to a pregnant woman, there are several naturally occurring adverse outcomes to consider. For example, miscarriages, stillbirths, and congenital malformations can occur without explanation in the general population. In addition, also consider the specific health history of the mother and the available research literature regarding the specific psychotropic agent (keeping in mind that there are ethical issues associated with conducting prospective research in pregnant women, such as it being unethical to withhold treatment to pregnant women who are depressed in order to have a control group, and that retrospective research is often confounded by recall bias). Potential risks to be aware of include miscarriage (spontaneous abortion), malformation (teratogenesis, birth defects), preterm delivery, neonatal adaptation syndrome, and behavioral teratogenesis (Table $\left.\mathbf{1}^{3-8}\right)$.

Selective serotonin reuptake inhibitors (SSRIs), the usual medication treatment of choice for depression, have at times been implicated in adverse pregnancy outcomes, but no strong evidence suggests they increase the miscarriage rate. Overall data are reassuring regarding the risk of malformation associated with SSRI use. Of note,

\section{Table 1}

\section{Potential negative outcomes of pregnancy (+/- medication)}

\begin{tabular}{|l|}
\hline $\begin{array}{l}\text { Potential negative outcomes of mental } \\
\text { illness occurring in pregnancy }\end{array}$ \\
\hline Relapse of mental illness \\
\hline Relapse of substance abuse \\
\hline Poor social functioning \\
\hline Suicide \\
\hline Violence perpetration \\
\hline Increased risk of intimate partner violence \\
\hline Accidental self-harm \\
\hline Poor prenatal care \\
\hline Preterm delivery \\
\hline Low birthweight \\
\hline
\end{tabular}

Potential negative outcomes of pregnancy with specific medications

Malformation (teratogenesis, birth defect) (eg, atrial septal defect, PPHN)

Miscarriage (spontaneous abortion)

Preterm delivery (with low birth weight)

Neonatal adaptation syndrome (toxicity or withdrawal; self-limited, transient symptoms such as disrupted sleep, jitteriness, irritability; related to overstimulation or withdrawal of serotonin)

Behavioral teratogenesis (later developmental problems such as developmental delay, autism, lower intellectual functioning, delayed neuromuscular development)

PPHN: persistent pulmonary hypertension in the neonate Source: References 3-8

the FDA had switched paroxetine from a Class C drug to a Class D drug after early reports of a potential $1.5 \%$ to $2 \%$ risk of fetal cardiac malformations compared with a $1 \%$ baseline risk in the general population (these FDA pregnancy risk letter categories have since been phased out). ${ }^{9,10}$ Nevertheless, the absolute risk remains small. Another large study found that there was no substantial increased risk of cardiac malformations attributable to antidepressant use during the first trimester. ${ }^{11}$

\section{Lessons from a class action suit}

Since we last reviewed pregnancy and antidepressants in 2013, 8 several class action lawsuits against the manufacturers of psychotropic medications have been heard.

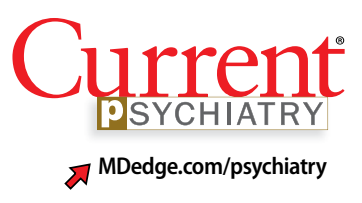

Clinical Point

Appropriate treatment of depression is even more important during pregnancy than at other times of life

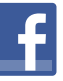

Discuss this article at www.facebook.com/ MDedgePsychiatry 


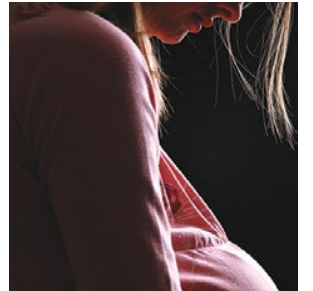

Depression during pregnancy

\section{Clinical Point \\ Overall data are reassuring regarding the risk of malformation associated with SSRI use}

\section{Table 2}

\section{Summary of key points from the Zoloft lawsuit and basis for Daubert challenge}

Challenged notion of class effect, noting that the FDA had separate warnings for paroxetine from the other SSRIs (Class D vs Class C) (acceptability, and methodology for potential meta-analysis)

Highlighted conflicting results of studies (not settled or generally accepted)

Highlighted that some earlier database studies that had reported statistical significance were not reproducible when included in larger analysis populations (reproducibility of results)

Challenged that post hoc meta-analysis done by expert after being retained had sampling biases (concerns over methodology)

Pointed out general factual errors/mistakes in one expert's data (concerns over methodology and reproducibility)

Demonstrated that some findings were not reproducible by other experts using the same data set (reproducibility)

Raised concerns in general that there may have been "cherry picking" of studies when the scientific method uses analysis of reasonable reliable data, whether pro or con (general accepted approach to scientific process)

Experts' limited ability to justify why some studies were considered more reliable than others (aspect of general acceptability)

SSRIs: selective serotonin reuptake inhibitors

Source: References 12,13

Product liability actions brought against manufacturers are different from medical malpractice suits brought against individual physicians, which may result from lack of informed consent, suicide, or homicide.

One of the largest class action suits was against Zoloft (specifically Zoloft and Pfizer, since the brand manufacturer is responsible for the product insert information. $)^{12,13}$ At the time, sertraline was already commonly prescribed due to the relatively safe reproductive profile.

Many of the more than 300 federal claims were united in a multi-district litigation (MDL) suit under the United States District Court of Eastern Pennsylvania (MDL 2342). Pfizer issued Daubert challenges (efforts to exclude the introduction of "junk science" into the courtroom) against the plaintiffs' experts' scientific methods and results. ${ }^{12,13}$ The plaintiffs (those suing Pfizer) had to prove that the medications caused the negative outcome, not that they were merely temporally associated. Subsequently, 2 plaintiff experts - a PharmD and a biostatistician-were removed. Pfizer successfully challenged the methodological soundness of the plaintiffs' experts' testimony (Table $2^{12,13}$ ), and the case was dismissed. In general, the courts identified the Bradford Hill criteria as often being important (though not definitive) methodology for determining causation (Table 3, ${ }^{12,13}$ page 33).

A concept raised in prior psychotropic lawsuits was the "learned intermediary doctrine," in which pharmaceutical companies stated that once a risk is known, it is the responsibility of the prescribing physician to assess risks vs benefits and inform the patient. ${ }^{8}$ Many aspects of the larger class action lawsuits related to failure of the company to do adequate research to identify risks and appropriately inform the public and the medical community of these risks. ${ }^{14}$

\section{Challenges in interpreting the literature}

Some of the difficulties in interpreting the literature on the association of antidepressants and birth defects can be seen in a 2020 study by Anderson et al. ${ }^{15}$ This study was published in JAMA Psychiatry, received widespread coverage in the media, and was discussed on the CDC's website. ${ }^{16}$ Anderson et $\mathrm{al}^{15}$ compared a large cohort of 30,630 infants with birth defects from the multicenter case-control National Birth Defects Prevention Study with 11,478 
randomly selected controls with no defects. Three primary study groups were women whose pregnancies resulted in:

- birth defects with no antidepressant exposure ( $n=28,719)$

- birth defects with exposure to an antidepressant $(\mathrm{n}=1,911)$

- no birth defect control group ( $\mathrm{n}=$ 10,886 no antidepressant exposure, $n=592$ antidepressant exposure).

This study reported there were "some associations between maternal antidepressant use and specific birth defects" and "Venlafaxine was associated with more birth defects than other antidepressants, which needs confirmation." 15 However, in an accompanying editorial, Wisner et $\mathrm{al}^{17}$ discussed potential problems and limitations with this study and research of this nature in general (Table $4,^{17}$ page 34). In addition, Anderson et $\mathrm{al}^{15}$ used certain "controversial" statistical practices. ${ }^{18}$ For example, "[T]o align with American Statistical Association guidelines to consider effect sizes when interpreting results instead of statistical significance, we noted associations as meaningfully elevated if [adjusted odds ratios] were 2.0 or greater and lower confidence interval bounds were 0.8 or greater." ${ }^{\prime 15}$

Those who read only abstracts or news stories may believe this study of $>40,000$ participants included a large number of women who were receiving venlafaxine. However, the number of pregnant women who were prescribed venlafaxine was actually very small-112 who took venlafaxine experienced a birth defect. In addition, the authors noted "Venlafaxine was associated with many of the same defects across the samples (data not shown)."15 As discussed above, historically one of the areas the courts have considered was whether or not appropriate methodology was applied, and whether the results could be replicated with the data provided.

Further, new studies need to be considered in context of the literature as a whole and collective clinical experience. A recent systematic review found that among 3,186 infants exposed to venlafaxine during the first trimester, there were 107 major malformations..$^{19}$ This indicated a relative risk

\section{Table 3}

\section{Summary of Bradford Hill criteria for causation $^{a}$}

Strength of association between medication exposure and concerned outcome

Temporal relationship

Dose-response relationship

Ability to replicate findings

Biologic plausibility of mechanism

Alternative explanations lacking

Specificity of association

Overall consistency with other scientific knowledge

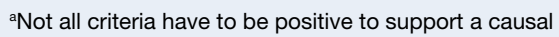
relationship

Source: References 12,13

estimate of 1.12 , with a $95 \%$ CI of 0.92 to 1.35. The authors concluded that venlafaxine exposure in the first trimester was not associated with an increased risk of malformations.

Expectant parents may come across a headline that implies a specific antidepressant causes problems, but have not read the study or know how to interpret it. Often it is best for a physician to find out what the basis of the concern is, and if possible, review the study with the patient to make sure it is in the right context, and if it applies to the individual patient's situation.

\section{Consider the ethical issues}

In addition to preventive ethics, other critical ethical issues in pregnancy include omission bias, beneficence, and autonomy., ${ }^{4,20-24}$ Omission bias occurs when physicians are more concerned about acts of commission (in which treatment leads to a negative outcome) than they are about acts of omission, which involve not treating the patient's illness. To address this, it is important to discuss with the patient both the risks of treating and the risks of not treating maternal depression, so that the mother can make the best decision for her own specific set of circumstances.

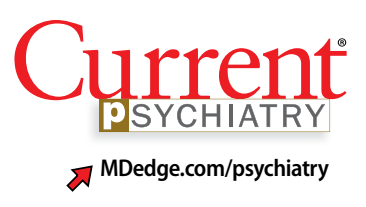

Clinical Point

New studies need to be considered in the context of the literature as a whole and collective clinical experience 


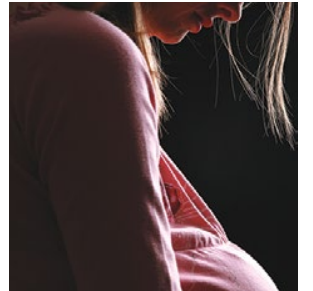

Depression during pregnancy

\section{Clinical Point}

When possible, monotherapy with the lowest possible effective dose is often the judicious approach to treatment
Regarding beneficence (promoting the patient's best interest), consider both the mother's and the infant's best interest, which usually are quite closely related. Women may feel guilty about taking a medication that they perceive is harmful for the fetus but good for their own mental health. Physicians can help with this by providing education about the benefits of treating depression for the fetus' benefit as well. The fetus is completely dependent on the environment that the mother places them in, not merely the medication effects (eg, psychologic/physiologic stress effects, poor diet, lack of exercise, risk of "self-medication").

Regarding autonomy (a woman's own decision-making), Coverdale et $\mathrm{al}^{21}$ discussed strategies that can enhance a pregnant patient's autonomy-including discussing treatment options and counselling about the effects of depression itself in pregnancy, as well as considering the effects of depression on the process of decision-making. For example, a woman with depression may see the world through a negative lens or may have difficulty concentrating. Patients may also require education about the concept of relative risk in comparison to absolute risk-especially in light of attention-grabbing headlines.

Finally, as part of preventative ethics, anticipate the ethical dilemmas before the common situation of pregnancy. Almost one-half of pregnancies are unplanned. ${ }^{25}$ Many women thus expose their fetus to medication during the critical early period of organogenesis, before noticing they were pregnant. Therefore, even if a patient of childbearing age insists that she is not sexually active, the prudent psychiatrist should still begin discussions about medications in pregnancy.

\section{An outline of best practices}

Best practice includes preventive ethics, and when treating any woman of childbearing age, psychiatrists should consider prescribing medications that are known to be relatively safe in pregnancy rather than risky in pregnancy. Therefore, any psychiatrist whose practice includes women of childbearing age should have a working knowledge of which agents are relatively safe in pregnancy. After a woman is pregnant, careful decision-making about medication should continue. Consult with reproductive psychiatry colleagues where necessary.

A patient with depression would usually merit closer follow-up during the pregnancy. In some cases, psychotherapy alone can be effective in depression. However, approximately $6 \%$ to $13 \%$ of women are prescribed antidepressants during pregnancy, and this has been increasing. ${ }^{26}$ Women who discontinue their antidepressant while pregnant are more likely to relapse than those who continue their medication, ${ }^{27}$ thus exposing their fetus to negative effects of depression as well as medication (prior to discontinuation). 
When possible, monotherapy (one agent) in the lowest effective dose is often the judicious approach to treatment. For a patient prescribed pre-existing polypharmacy at time of pregnancy, a risk-benefit analysis of which medications should remain, which should be stopped, and a plan for taper, if needed, should be discussed and documented. Using too little of an antidepressant dose would expose the fetus to both depression and medication, whereas using a maximum dose when not needed would expose the fetus to more medication than is necessary to treat the mother's symptoms. This discussion with the mother (and her partner, if available) should be documented in the chart. The mother should understand both the risk of untreated illness and the potential risks of medications, as well as the benefits of medications and alternatives. It is important for the mother to realize that there is no risk-free option, and that malformations can occur in the general population as well as in individuals with untreated depression, separate from any medication exposure. In fact, most malformations do not have a known cause, and overall approximately $3 \%$ of pregnancies result in a birth defect. ${ }^{28}$

If possible, discuss the treatment plan with the patient's obstetrician, or ask the mother to discuss the plan with her obstetrician, so that everyone is on the same page. This discussion can help attenuate patient anxiety that results from hearing different things from different clinicians. Communication with other treating professionals (eg, OB/GYNs, pediatricians) can be beneficial and reduce liability if multiple physicians have agreed on a treatment plan-even if there is a negative outcome. With malpractice, a clinician is not necessarily at fault for a bad outcome or adverse effect, but is at fault for lack of informed

\section{Related Resources}

- MotherToBaby. www.mothertobaby.org/

- Centers for Disease Control and Prevention. Treating for two: medicine and pregnancy. www.cdc.gov/pregnancy/ meds/treatingfortwo/index.html

- MGH Center for Women's Mental Health. Reproductive psychiatry resource and information center. www. womensmentalhealth.org/

Drug Brand Names

Paroxetine - Paxil

Sertraline $\cdot$ Zoloft

Venlafaxine - Effexor

consent or negligence (deviation from standard of care), which is harder for an attorney to demonstrate if there is deliberation, communication, and a plan that multiple doctors agree upon.

Be aware that informed consent is an ongoing process, and a woman may need to be reminded or informed of potential risks at varying stages of her life (eg, when starting a new relationship, getting married, etc.). Documentation can include that the clinician has discussed the risks, benefits, adverse effects, and alternatives of various medications, and a description of any patient-specific or medication-specific issues. In addition to verbal discussions, giving patients printed information can be helpful, as can directing them to appropriate websites (see Related Resources). Some physicians require patients to sign a form to indicate that they are aware of known risks.

Similar to being proactive before your patient becomes pregnant, think proactively regarding the postpartum period. Is your patient planning to breastfeed? Is the medication compatible with breastfeeding, or is bottle feeding the best option considering the mother's specific circumstances? For example, developing severe symptoms, experiencing insomnia, needing to take

\section{Bottom Line}

Concerns about being sued should not prevent appropriate care of depression in a woman who is pregnant. Discuss with your patient both the risk of untreated mental illness and the risk of medications to ensure she understands that avoiding antidepressants does not guarantee a safe or healthy pregnancy. 


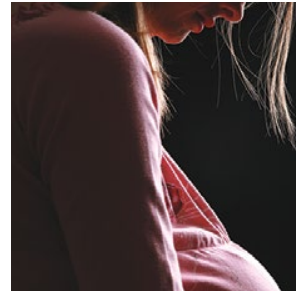

\section{Depression during} pregnancy

\section{Clinical Point}

Informed consent is an ongoing process, and a woman may need to be reminded of potential risks at varying stages of life a contraindicated medication, or having a vulnerable infant might sway a mother towards not breastfeeding. The expectant mother (and her partner, where possible) should be educated about postpartum risks and the importance of sleep in preventing postpartum depression.

\section{References}

1. Palmsten K, Setoguchi S, Margulis AV, et al. Elevated risk of preeclampsia in pregnant women with depression: depression or antidepressants? Am J Epidemiol. 2012; 175(10):988-997.

2. Sit DK, Perel JM, Helsel JC, et al. Changes in antidepressant metabolism and dosing across pregnancy and early postpartum. J Clin Psychiatry. 2008;69(4):652-658.

3. Grote NK, Bridge JA, Gavin AR, et al. A meta-analysis of depression during pregnancy and the risk of preterm birth, low birth weight, and intrauterine growth restriction. Arch Gen Psychiatry. 2010;67(10):1012-1024

4. Friedman $\mathrm{SH}$. The ethics of treating depression in pregnancy. J Prim Health Care. 2015;7(1):81-83.

5. Friedman SH, Resnick PJ. Postpartum depression: an update. Women's Health. 2009;5(3):287-295.

6. Liu Y, Kaaya S, Chai I, et al. Maternal depressive symptoms and early childhood cognitive development: a metaanalysis. Psychol Med. 2017;47(4):680-689.

7. Wisner KL, Sit DK, Hanusa BH, et al. Major depression and antidepressant treatment: impact on pregnancy and neonatal outcomes. Am J Psychiatry. 2009; 166(5): 557-566.

8. Friedman $\mathrm{SH}$, Hall RCW. Antidepressant use during pregnancy: How to avoid clinical and legal pitfalls. Current Psychiatry. 2013;12(2):21-25.

9. Bar-Oz B, Einarson T, Einarson A, et al. Paroxetine and congenital malformations: meta-analysis and consideration of potential confounding factors. Clin Ther. 2007;29(5): 918-926.

10. Einarson A, Pistelli A, DeSantis M, et al. Evaluation of the risk of congenital cardiovascular defects associated with use of paroxetine during pregnancy. Am J Psychiatry. 2008;165(6):749-752

11. Huybrechts KF, Palmsten K, Avorn J, et al. Antidepressant use in pregnancy and the risk of cardiac defects. N Engl J Med. 2014;370(25):2397-2407.

12. In re: Zoloft (sertraline hydrochloride) products liability litigation. MDL No. 2342. No. 12-md-2342. United States District Court, E.D. Pennsylvania. June 27, 2014.

13. In re: Zoloft (sertraline hydrocloride) products liability litigation. MDL No. 2342. United States District Court, E.D. Pennsylvania. December 2, 2015.
14. Kirsch N, Pacheco LD, Hossain A, et al. Medicolegal review: perinatal Effexor lawsuits and legal strategies adverse to prescribing obstetric providers. AJP Rep. 2019;9(1):e88-e91.

15. Anderson KN, Lind JN, Simeone RM, et al. Maternal use of specific antidepressant medications during early pregnancy and the risk of selected birth defects. JAMA Psychiatry. 2020;77(12):1246-1255.

16. Centers for Disease Control and Prevention. Use of the antidepressant venlafaxine during early pregnancy may be linked to specific birth defects. Published October 28 2020. Accessed October 29, 2020. https:/ /www.cdc.gov/ ncbddd/birthdefects / features/venlafaxine-duringpregnancy.html

17. Wisner KL, Oberlander TF, Huybrechts KF. The association between antidepressant exposure and birth defects--are we there yet? JAMA Psychiatry. 2020;77(12): 1215-1216

18. Wasserstein RL, Lazar NA. The ASA statement on $p$-values: context, process, and purpose. American Statistician. 2016; 70(2):129-133

19. Lassen D, Ennis ZN, Damkier P. First-trimester pregnancy exposure to venlafaxine or duloxetine and risk of major congenital malformations: a systematic review. Basic Clin Pharmacol Toxicol. 2016;118(1):32-36.

20. Miller LJ. Ethical issues in perinatal mental health. Psychiatr Clin North Am. 2009;32(2):259-270.

21. Coverdale JH, McCullough JB, Chervenak FA. Enhancing decision-making by depressed pregnant patients. J Perinat Med. 2002;30(4):349-351

22. Coverdale JH, McCullough LB, Chervenak FA, et al. Clinical implications of respect for autonomy in the psychiatric treatment of pregnant patients with depression. Psychiatr Serv. 1997;48:209-212.

23. Coverdale JH, Chervenak FA, McCullough LB, et al Ethically justified clinically comprehensive guidelines for the management of the depressed pregnant patient. Am J Obstet Gynecol. 1996;174(1):169-173.

24. Wisner KL, Zarin DA, Holmboe ES, et al Risk-benefit decision making for treatment of depression during pregnancy. Am J Psychiatry. 2000;157(12):1933-1940.

25. Finer LB, Zolna MR. Unintended pregnancy in the United States: incidence and disparities, 2006. Contraception. 2011:84(5):478-485.

26. Cooper WO, Willy ME, Pont SJ, et al. Increasing use of antidepressants in pregnancy. Am J Obstet Gynecol. 2007;196(6):544.e1-5.

27. Cohen LS, Altshuler LL, Harlow BL, et al. Relapse of major depression during pregnancy in women who maintain or discontinue antidepressant treatment. JAMA. 2006;295(5):499-507.

28. Centers for Disease Control and Prevention. Update on overall prevalence of major birth defects--Atlanta, Georgia, 1978-2005. MMWR Morb Mortal Wkly Rep. 2008;57(1):1-5. 